\title{
Efecto del conformado en frío en la sinterización de nano-polvos de alúmina
}

\author{
A. ARELLANO', J. LEMUS-RUIZ1', D. BOUVARD², S. DíAZ DE LA TORRE³, L. OLMOS \\ 'Instituto de Investigaciones Metalúrgicas, UMSNH \\ ${ }^{2} \mathrm{SiMaP}$, Institut Polytechnique de Grenoble \\ ${ }^{3}$ CIITEC, Instituto Politécnico Nacional \\ ${ }^{4}$ Coordinacion de la Investigación Científica, UMSNH
}

\begin{abstract}
El presente trabajo se enfoca a estudiar efecto del proceso de conformado en la transformación y evolución de la microestructura de alúmina durante el sinterizado convencional de polvos esféricos de $\gamma$-alúmina con tamaño promedio de partícula de $35 \mathrm{~nm}$. Las muestras fueron preparadas por tres diferentes rutas de conformado; colada por barbotina, prensado axial e isostático. Los compactos fueron sinterizados a $1500{ }^{\circ} \mathrm{C}$ durante una hora en un dilatómetro vertical. Las muestras fueron evaluadas usando MEB y DRX con el propósito de observar la microestructura final, el tamaño de grano se obtuvo por análisis de imágenes. Los resultados revelaron que el sinterizado es activado, inicialmente en los compactos hechos mediante prensado ya sea axial o isostático, sin embargo, la máxima densificación se obtiene en muestras fabricadas por colada continua. El tamaño de grano más pequeño fue obtenido cuando la compactación isostática fue utilizada. Por el contrario la evolución a la fase $\alpha$-alúmina se incrementa en muestras formadas por colada de barbotina.
\end{abstract}

Palabras clave: Sinterización, Prensado axial, Prensado isostático, Alúmina, Colada de barbotinas, Nano-polvos.

\section{Effect of the cold forming processes on the sintering of alumina nano-powders}

This work focused in the study of the effect of the conforming process on the transformation of the alumina during the conventional sintering of spherical $\gamma$-alumina powders with a $35 \mathrm{~nm}$ of the average particle. We made compacts by three different method; slip casting, axial and isostatic compaction. Then sintering was carried out at $1500{ }^{\circ} \mathrm{C}$ with an isothermal plateau of 1 hour in a vertical dilatometer. Samples were evaluated by SEM and XRD in order to observe the final microstructures, the final grain size was assessed by image analysis. Results show that sintering was activated earlier for the compacts produced by compaction either axial or isostatic, however maximal densification was observed for the slip casting. The smaller final grain size was obtained when isostatic compaction is used. In the other hand the evolution into the $\alpha$-alumina was promoted by the slip casting.

Keywords: Sintering, Axial compaction, Isostatic compaction, Alumina, Slip casting, Nano-powders.

\section{INTRODUCCIÓN}

La fabricación de polvos de tamaño nanométrico puede realizarse de manera industrial, sin embargo, el procesamiento para convertirlos en materiales sólidos conservando la nanoestructura es todavía un reto. Varios parámetros influyen en la evolución microestructural durante la sinterización, como; la temperatura, presión, tamaño de partícula y atmósfera ${ }^{(1-4)}$. Así, la síntesis y empaquetamiento de los polvos, han mostrado tener una influencia durante el sinterizado. La alúmina es el material cerámico más estudiado, no obstante, presenta cambios polimórficos que hace difícil determinar los mecanismos de crecimiento de grano $^{(5-7)}$. Mazaheri y colaboradores ${ }^{(8)}$ utilizaron dos tipos de conformado en verde, prensado axial e isostático, y encontraron que a pesar de tener una densidad en verde uniforme el crecimiento de grano se produce en forma inminente; por lo que utilizaron dopantes para evitar el crecimiento de grano durante el sinterizado, reduciéndolo a $35 \mathrm{~nm}$. Posteriormente, Azar y colaboradores ${ }^{(9)}$ demostraron que el método de conformado es crucial para la transformación de fase, obteniendo que la colada por barbotina favorece la transformación de fases y densificación en comparación con el prensado axial. Por su parte, Bowen y colaboradores ${ }^{(10)}$ ratificaron que la densificación obtenida por colada por barbotina es mayor pero también el tamaño de grano final. Palmero ${ }^{(11)}$ propuso que el crecimiento vermicular generado por las alúminas de transición es perjudicial para retener la nanoestructura y comprobó que a elevadas temperaturas se puede evitar. En el presente trabajo se estudia el efecto del método de conformado de nano-polvos de $\gamma$-alúmina durante el sinterizado convencional a elevada temperatura. 


\section{PARTE EXPERIMENTAL}

En el presente trabajo se usaron polvos comerciales de $\gamma-\mathrm{Al}_{2} \mathrm{O}_{3}(99.99 \%)$, TAIMICRON TM50. Algunas propiedades físicas de los polvos de $\gamma-\mathrm{Al}_{2} \mathrm{O}_{3}$ son: área superficial de 68.2 $\mathrm{cm}^{2} / \mathrm{g}$, tamaño de partícula de $0.05 \mu \mathrm{m}$ con morfología esférica y fases cristalinas de $70 \gamma-30 \alpha$. Los polvos de $\gamma-\mathrm{Al}_{2} \mathrm{O}_{3}$ con tamaño nanométrico forman aglomerados en verde que en su interior coexisten porosidades inter-aglomeradas e intercristalinas, Figura 1, que durante el calentamiento se eliminan formando la típica microestructura vermicular de la alúmina favoreciendo al crecimiento de grano ${ }^{(11)}$.

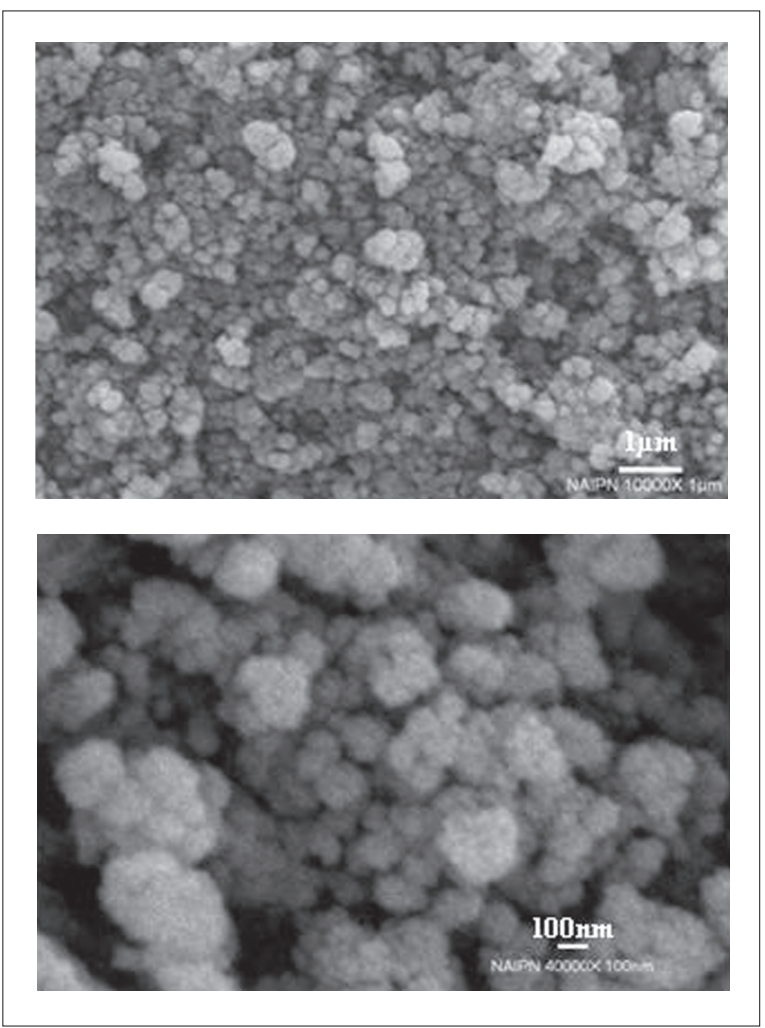

Figura 1. Micrografía de los polvos de $\gamma-\mathrm{Al}_{2} \mathrm{O}_{3}$ iniciales donde se observa la formación de los aglomerados debido a su tamaño nanométrico.

La distribución de tamaño de partícula se obtuvo a partir del área superficial y mediante análisis de imágenes, coincidiendo en un diámetro promedio de $35 \mathrm{~nm}$.

\subsection{Conformado de Polvos}

Los polvos fueron conformados por tres mecanismos en verde; colada de barbotina (CB), prensado axial (PA) y prensado isostático (PI). En CB los polvos, $\gamma-\mathrm{Al}_{2} \mathrm{O}_{3}$, deben permanecer en suspensión coloidal con $\mathrm{NH}_{4} \mathrm{OH}$ diluido en agua a una concentración de $0.01 \mathrm{~N}$ manteniendo un $\mathrm{pH}$ en 10 estabilizando con $\mathrm{HNO}_{3}$ debido a que no interviene en la evolución de las fases y se elimina a $85{ }^{\circ} \mathrm{C}$. A la solución se le agregan $2 \mathrm{~g}$ de polvos de $\gamma$-alúmina. La disminución de la viscosidad, así como el mejoramiento de la fluidez durante el vaciado se logra con un defloculante, Darvan C (poli-metacrilato de amonio) a una concentración de $0.03 \%$ en peso. La mezcla se homogeniza utilizando una tina de ultrasonido durante $1 \mathrm{~h}$ a $1500 \mathrm{~Hz}$. Finalmente, la suspensión se vertió en un molde de yeso cerámico de $0.8 \mathrm{~cm}$ de diámetro, dejándola secar durante $5 \mathrm{~h}$ antes de desmontar la pieza. Para el PA se utilizó un dado de acero de $0.8 \mathrm{~cm}$ de diámetro. Los polvos fueron sometidos a una presión axial de $400 \mathrm{MPa}$, en una prensa P/OWEBER. Por otro lado, para el PI se utilizaron dos prensas, la mencionada anteriormente para fabricar un compacto a una presión de $150 \mathrm{MPa}$ y una presión isostática en una prensa $A V U R E$ de canasta movible, usando aceite frio en su interior a una presión de $250 \mathrm{MPa}$. Para garantizar la uniformidad de la presión se utilizó un tiempo de estancia de 5 minutos a la presión final ejercida.

\subsection{Etapa de Sinterizado}

Previo a la sinterización y con el fin de eliminar los ligantes, a los compactos se les realizó un tratamiento térmico en un horno eléctrico horizontal calentando a $2.5{ }^{\circ} \mathrm{C} / \mathrm{min}$ en aire a $600{ }^{\circ} \mathrm{C}$ durante $2 \mathrm{~h}$. La sinterización se realizó en un dilatómetro vertical SETARAM/SETSYS 68/2, que permite medir la contracción/dilatación del material in-situ en función de la temperatura, calentando a $25^{\circ} \mathrm{C} / \mathrm{min}$ hasta $1500{ }^{\circ} \mathrm{C}$ en atmósfera de aire. Para cada compacto se calculó su densidad relativa geométrica antes y después del sinterizado. El análisis de transformación de fases se realizó por la técnica de difracción de rayos $\mathrm{X}$ en un rango angular $2 \theta$ de 10 a $100^{\circ}$ con una velocidad de barrido de $0.02^{\circ} / \mathrm{s}$ en un difractómetro SIEMENS 400 usando radiación de CuKa a $30 \mathrm{kV}$ y 25 mA. Para la observación de la morfología de los compactos obtenidos, estos fueron cortados y pulidos a espejo para su evaluación en un microscopio electrónico de barrido (MEB) LEO STEREOSCAN S440 a un voltaje de $1.5 \mathrm{Kev}$.

\section{RESULTADOS}

En la Figura 2a se presenta el comportamiento durante el ciclo de sinterización para los diferentes compactos en donde se puede apreciar que en los primeros $1000^{\circ} \mathrm{C}$ los tres métodos de conformado presentan la misma deformación, la cual es relacionada con la expansión térmica del material. A partir de los $1020^{\circ} \mathrm{C}$ en los conformados por prensado se activa la sinterización, la cual se denota por un cambio abrupto en la deformación. Por otro lado, en los compactos formados por barbotina el sinterizado se activa a $1120{ }^{\circ} \mathrm{C}$. Cuando inicia el sinterizado se observa una contracción de volumen hasta que comienza el cambio de fase, manifestado por un cambio en la pendiente de deformación. El cambio de fase para cada compacto se lleva a cabo a temperaturas diferentes en el rango de $1100-1250{ }^{\circ} \mathrm{C}$, lo cual es consistente con los reportes hechos por Bowen y colaboradores ${ }^{(10)}$. La temperatura de inicio del cambio de fase para compactos PA fue de $1130{ }^{\circ} \mathrm{C}$, para PI de $1170{ }^{\circ} \mathrm{C}$ y para $\mathrm{CB}$ de $1230{ }^{\circ} \mathrm{C}$. A $1500{ }^{\circ} \mathrm{C}$ las muestras PI llegan a su máximo encogimiento después de 8 minutos, mientras que los compactos PA llegan en 10 minutos, estos tiempos indican que la mayor densificación se lleva a cabo durante el calentamiento, iniciando la sinterización a $1020^{\circ} \mathrm{C}$. Por otro lado, los resultados de los compactos CB requieren más tiempo para su máximo encogimiento, debido a la forma de empaquetamiento de las partículas. La velocidad de densificación varia con respecto al tipo de conformado, Figura 2b, los conformados por PI presentan la máxima velocidad de los tres compactos antes del cambio de fase, lo 
que permite que el compacto logre un $38 \%$ de su densificación total, en ese periodo. Los compactos PA muestran una menor densificación que los PI pero mayor que los CB y logran 25 $\%$ de su densificación total antes del cambio de fase. Para los conformados por CB la velocidad es la más baja durante esta etapa y solo logran un $15 \%$ de su densificación total. Al comparar las tres gráficas, los conformados por PI y PA llegan a su máxima velocidad de densificación antes del cambio de fase; sin embargo para CB alcanza su máxima velocidad a la mitad del ciclo térmico después del cambio de fase.

En la Figura 3 se presentan los resultados de difracción de rayos $\mathrm{X}$ para las diferentes muestras después del sinterizado, así como de los polvos iniciales. Se observa que en los polvos iniciales coexisten las fases $\gamma-\alpha$. El pico a $20^{\circ}$ es característico de la fase Gibbsita ( $\gamma$-alúmina) y el pico a $43^{\circ}$ pertenece a la fase

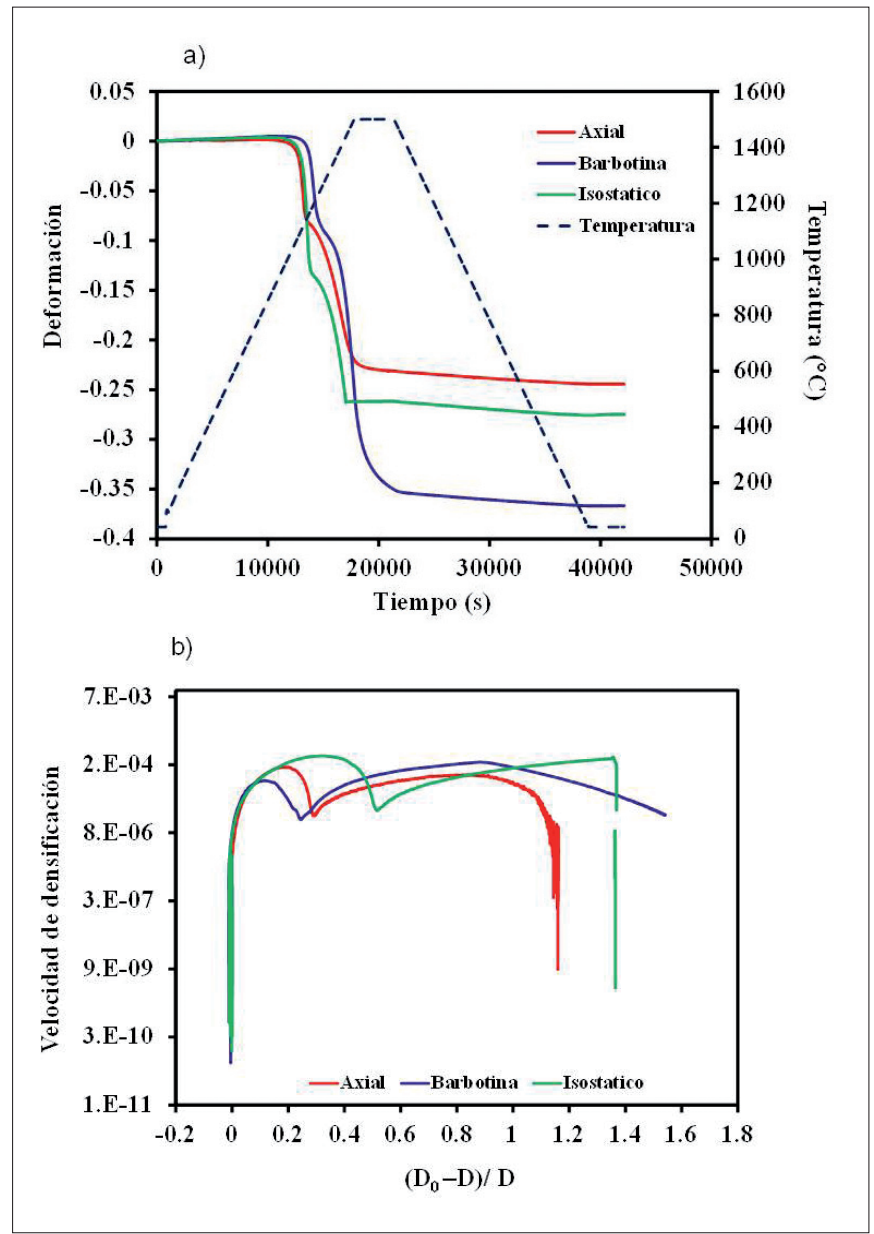

Figura 2. Comportamiento durante el sinterizado para los diferentes conformados en frio utilizados: a) efecto del tiempo en la deformación y b) velocidad de deformación. estable $\alpha$. Después del sinterizado, las muestras PA denotan la evolución de la microestructura por un ensanchamiento del pico a $20^{\circ}$ con un desplazamiento en ángulos bajos, lo que indica la formación de una fase amorfa conocida como pseudo-boehmita. Esto se desarrolla por deshidratación del trióxido de aluminio de la Gibbsita; también se observa que en este conformado se presentan reflexiones difusas en el rango de $10-20^{\circ}$ lo que establece que en prensado axial no transforma la fase totalmente aún llegando a la temperatura de sinterización. Por otro lado, en los PI se nota la definición de los picos de la fase estable $\alpha-\mathrm{Al}_{2} \mathrm{O}_{3^{\prime}}$ es decir, la cristalinidad aumenta con respecto a las PA pero todavía no transforma por completo la fase. El pico a $26^{\circ}$ pertenece a la fase $\gamma-\mathrm{Al}_{2} \mathrm{O}_{3} \mathrm{y}$ para el pico a $77^{\circ}$ la división que se observa indica que sigue transformando la fase. Para los CB los picos principales de la fase estable están bien definidos, teniendo una intensidad mayor, comparando con los PA y PI. La fase estable alfa se comprueba en los picos de $26^{\circ}, 35^{\circ} 43^{\circ}, 57^{\circ}, 67^{\circ}$ y $69^{\circ}$, presentes en los conformados por PI y CB. La transformación de fase para PA es de $60 \%$, para PI $87 \%$ y para CB $100 \%$.

La Figura 4 muestra la microestructura interna de los compactos después del sinterizado, observada por MEB. Se enfatiza que cada método de conformado generó un incremento en el crecimiento de grano diferente durante el mismo ciclo de sinterizado. El tamaño de grano fue estimado mediante el análisis de imágenes encontrando un tamaño promedio de $2.3 \mu \mathrm{m}, 1 \mu \mathrm{m}$ y $1.8 \mu \mathrm{m}$ para PA, PI y CB, respectivamente. La porosidad más grande se encontró en muestras PA la cual se encuentra dentro de los granos y es muy difícil de eliminar,

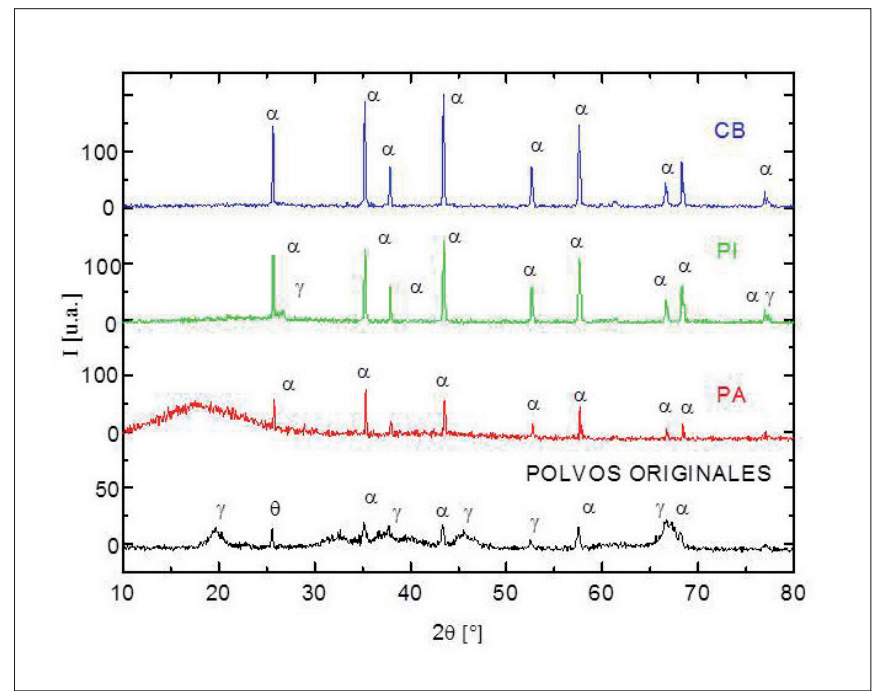

Figura 3. Difractogramas de los compactos sinterizados con diferentes procedimientos de conformado en frio.
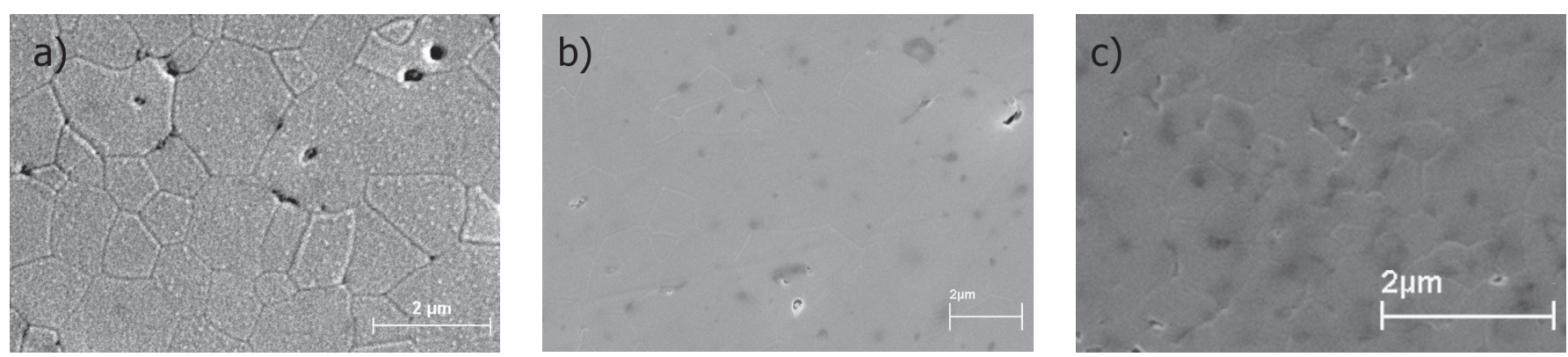

Figura 4. Micrografías de la microestructura interna de las muestras sinterizadas a $1550^{\circ} \mathrm{C}$, a) PA, b) PI y c) CB. 
Figura 4a, a diferencia de las muestras PI ó CB donde los poros son más pequeños y en su mayoría se encuentran en los límites de grano, Figura $4 \mathrm{~b}$ y $4 \mathrm{c}$, respectivamente.

\section{DISCUSIÓN}

Se encontró que el método de conformado tiene un efecto sobre el comportamiento del sinterizado de nano-polvos de $\gamma-\mathrm{Al}_{2} \mathrm{O}_{3}$. El conformado afecta la activación del sinterizado y la transformación de fase. Es claro, que el empaquetamiento de las partículas juega un papel importante en la evolución de las alúminas de transición $(\gamma-\delta-\theta)$ hasta llegar a la fase más estable, alfa, que se forma alrededor de los $1200{ }^{\circ} \mathrm{C}$. Para el caso del conformado por prensado, la fuerza de repulsión entre los cristales de las partículas es suprimida, por la presión externa, dejando una distancia nula entre partículas, lo contrario al CB que incrementa la distancia entre partículas. El efecto principal de la distancia de repulsión afecta a la transformación de fases intermedias, la cual es completa en CB generando un retardo en la activación del sinterizado, a diferencia de los conformados por prensado donde no se observa esta transformación. El inicio de la transformación final a la fase estable, alfa, se presenta a diferentes temperaturas y es asociada a la heterogeneidad de la distribución de densidad en cada compacto; se presume que los PA presentan una diferencia mayor entre la superficie cerca al punzón de prensado y el centro, lo cual genera que el calor sea transmitido de manera diferencial en todo el compacto. Esto impacta en el inicio de la transformación de fase en muestra PA, adelantando la temperatura de transición con respecto a la reportada por Legros y colaboradores ${ }^{(12)}$. Por otro lado, en el PI se homogeniza la distribución de densidades en todo el compacto, sin embargo esta distribución no es tan buena como en la $\mathrm{CB}$, observándose el inicio de la transformación de fase a una temperatura más cercana a la reportada.

El tipo empaquetamiento afectan tanto la transición de las fases como el crecimiento de grano durante el ciclo térmico. Es decir, como la formación de los cristales de la fase $\theta$ a la fase alfa sucede rápidamente para producir las colonias de alfa, los cristales se alejan de la matriz $\theta$ para formar una red de canales de poros y dendritas. Sin embargo, la microestructura obtenida se compone de una red de canales de poros los cuales para eliminarlos se requiere un crecimiento de grano mayor que en PI. La densificación es una característica asociada directamente a la porosidad, la cual generada por la estructura vermicular de la alúmina; la cual se trata de evitar durante el calentamiento. Por otro lado, la evolución de las alúminas de transición puede resultar conveniente, ya que cuando una fase de menor importancia se encuentra activa, generan un mínimo de energía que es reducida de la energía total para el cambio de fase logrando así un tamaño de grano menor, en comparación de cuando se transforman por completo los cristales a la fase estable. Para los conformados PA al variar el calor, las partículas adquieren distintas energías haciéndolas migrar en diferente posición superficial aumentando la energía cinética y activando una depresión en la temperatura, lo cual provoca la formación de la estructura vermicular y por lo tanto se generan un número considerable de poros.

\section{CONCLUSIONES}

Se investigó el efecto que el conformado en frío tiene sobre la evolución microestructural de nano-polvos de alúminas de transición durante el sinterizado a $1500{ }^{\circ} \mathrm{C}$. Se encontró que en función del proceso de conformado el comportamiento de los polvos fue diferente para un mismo ciclo térmico. Dos efectos son observados, un cambio en las temperaturas de transformación y una evolución del tamaño de grano diferente. La CB presentó las mejores condiciones para la trasformación completa durante el ciclo térmico utilizado, mientras que los PI retrasaron el crecimiento de grano. El conformado por PA presenta las peores características finales de la muestra, con mayor porosidad y tamaño de grano. La evolución de la microestructura durante el sinterizado de nano-polvos es fuertemente influenciada por el proceso de conformado.

\section{AGRADECIMIENTOS}

Los autores agradecen al Consejo Nacional de Ciencia y Tecnología (CONACYT) proyecto CB-2011/167286 y a la Universidad Michoacana de San Nicolás de Hidalgo por el apoyo y facilidades para el desarrollo del presente trabajo.

\section{REFERENCIAS}

1. Y. Zhou, K. Hiaro, Y. Yamauchi, and S. Kanzaki. Densification and grain growth in pulse electric current sintering of alumina. J. Eur. Ceram. Soc. 24, $12,3465-70$ (2004)

2. S-J.L Kang Sintering.Ist ed.London: Elsevier Butterworth-Heineman (2005)

3. J. Li , Y. Ye. Densification and grain growth of $\mathrm{Al}_{2} \mathrm{O}_{3}$ nanoceramics During Pressureless Sintering. J. Amer. Ceram. Soc. 89, 1, 139-43 (2006)

4. A. Shiu, Z. Kato, S. Tanaka, N. Uchida, K. Uematsu, Sintering deformation caused by particle orientation in uniaxially and isostatically pressed alumina compact. J. Eur. Ceram. Soc. 27, 3, 311-16 (2002)

5. Y. Wang, C. Suryanarayana, L. An, Phase Transformation in NanometerSized $\gamma$-Alumina by Mechanical Milling . J. Amer. Ceram. Soc 88, 3, 780-83 (2005)

6. H-C. Kao, W-C. Wei, Kinetics and Microstructural Evolution of Heterogeneous Transformation of $\theta$-Alumina to $\alpha$-Alumina. J. Amer. Ceram. Soc 83, 2, 362-68 (2000)

7. J.G. Li, X. Sun, Synthesis and sintering behavior of a nanocrystallite $\alpha$-Alumina powder. Acta Mater. 48, 3103-112 (2000)

8. M. Mazaheri, A. Simchi, F. Golestani-Fard, Densification and grain growth of nanocrystalline 3 Y-TZP during two-step sintering. J. Eur. Ceram. Soc. 28, 2933-39 (2008)

9. M. Azar, P. Palmero, M. Lombardi, V. Garnier, L. Montanaro, Effect of initial particle on the sintering of nanostructures transition alumina. J. Eur. Ceram. Soc. 28, 1121-28 (2008)

10. P. Bowen, C. Carry, D. Luxemburgo, H. Hofmann, Colloidal processing and sintering of nanosized transitions aluminas. Powder Tech. 157, 100-07 (2005)

11. P. Palmero, M. Lombardi, L Montanaro, Effect of heating rateo $n$ phase and microstructural evolution during pressureless sintering of a nanostructured transition alumina. J. Appl. Ceram. Tech. 6, 3, 420-30 (2009)

12. C. Legros, C. Carry, P. Bowen, H. Hofmann, Sintering of transition Alumina: Effects of phase transformation, powder characteristics and thermal cycle. J. Eur. Ceram. Soc. 19, 1967-78 (1999) 